\title{
PARAMETER IDENTIFICATION OF HYDRO-MECHANICAL PROCESSES USING ARTIFICIAL INTELLIGENCE SYSTEMS
}

\author{
Ivan Pavlenko ${ }^{1}$, Justyna Trojanowska ${ }^{2}$, Vitalii Ivanov ${ }^{3}$, Oleksandr Liaposhchenko $^{4}$ \\ 1,3,4 Sumy State University, 2 Rymskogo-Korsakova St., Sumy 40007, Ukraine \\ 2Poznan University of Technology, 3 Piotrowo Street, 60-665 Poznan, Poland \\ i.pavlenko@omdm.sumdu.edu.ua, justyna.trojanowska@put.poznan.pl, \\ ivanov@tmvi.sumdu.edu.ua,o.liaposhchenko@pohnp.sumdu.edu.ua
}

\begin{abstract}
The article is aimed at developing the comprehensive approach of parameter identification of complicated hydro-mechanical system by means of artificial intelligence systems (AIS) using artificial neural networks (ANN) and genetic algorithms (GA). The scientific novelty of the proposed method is inconsequent implementation of numerical analysis approach (e. g., FEM and CFD simulations), mathematical modeling of hydro-mechanical processes using the quasilinear regression procedure (QLRP), and artificial intelligence systems. Algorithms and related design schemes for implementation of the abovementioned technique are proposed for solving the interdisciplinary problems of aero-elastic interaction of gas-dispersed flow with the previously deformed flexible plates in a separation channel, static calculations of fixtures for parts manufacturing, as well as dynamic analysis of centrifugal compressors' rotors. All the presented has a significant advantage in comparison with the direct solving of the abovementioned problems. This advantage is in the ability of the comprehensive sequence "FEM / CFD - ANN / GA - QLRP" to predict the solutions of highly nonlinear mathematical models describing hydro-mechanical processes with uncertainties. As a result, unknown parameters of the mathematical models describing the complicated hydro-mechanical interactions are refined under an incompleteness of the initial data.
\end{abstract}

Keywords: Separation Equipment, Fixture, Finite Element Method, Artificial Neural Network, Genetic Algorithm, Regression Procedure.

\section{Introduction}

Artificial intelligence systems were firstly used for modeling the human nervous systems. However, nowadays they can be implemented as a universal numerical technique for solving applied problems in the field of computer engineering [1], designing control systems [2], as well as optimization of manufacturing processes $[3,4,5]$. Particularly, ANN models and related designing approaches are in the paper [6]. From this point of view, ANN can be applied as a simplified and modified model of brain activity. The wide use of these systems in the theory of recognition and to predict the big data is based on their advantages in comparison with the traditional approaches. Particularly, it should be noted, that ANN allows solving problems after the training by empiric data [7]. Moreover, a row of recent publications in the field of solving multidisciplinary problems is available $[8,9,10]$.

Nevertheless, there are no significant advances in the use of artificial intelligence systems for solving applied problems in the field of mechanical engineering due to the absence of unified approaches for their implementation. Moreover, recent research works are significantly varied in ANN implementations in the fields of mechanical, manufacturing and chemical engineering.

Due to the abovementioned, this paper is aimed at the generalization of the procedure of solving a wide range of applied problems in the field of engineering. This aim is reached for solving the direct and inverse problems of parameter identification. As a result, using comprehensive sequence "finite element modeling - artificial neural network - regression procedure" is proposed to evaluate the parameters of nonlinear mathematical models describing hydro-mechanical processes. Finally, all the research problems can be divided into conducting the directional research using ANN as the generalized regression procedure, as well as solving the inverse problem for parameter identification of the reliable mathematical models.

\section{Literature Review}

There are a lot of recent research works aimed at providing nonlinear regression analysis and developing fundamentals of using artificial intelligence systems and their applications. 
Particularly, a linear regression method based on genetic algorithm is presented in the research paper [11], and the related applications in the fields of engineering and computational mathematics are proposed. An effective procedure for the system prediction based on the quasilinear regression model is proposed in the paper [12], and related examples for combined using QLRM and GA are presented.

The research paper [13] is aimed at analyzing the characteristic and weakness of the existing regression methods. As a result, the parameter estimation strategy for quasilinear regression method is considered, as well as parameter estimation based on genetic algorithm and the least squares method is proposed. The presented example allowed concluding that the proposed approach is relatively good operable and can be widely used in many fields, including artificial intelligence. The research work [14] is aimed at developing a comprehensive approach for combined using artificial intelligence and probabilistic methods for mathematical analysis of physical that was numerically verified. Additionally, the probabilistic model-building genetic algorithm is proposed in the research work [15].

ANN can be more useful for solving directional research due to its ability to approximate obtained experimental results. The cooperation of many "neurons" of ANN performs the evaluated parameters. First significant attempts to implement ANN in the field of mechanical engineering were made in the work [16] for the case of continuous mechanical systems. The attempt of developing the scientific and methodological approaches for carrying out parameter identification of mathematical models of mechanical systems using artificial neural networks is presented in paper [17]. Additionally, the paper [18] is also devoted to the implementation of ANN for parameter identification of mathematical models describing the dynamic state of the mechanical system on the example of rotor dynamics. Another example of the application of ANN in engineering is presented in the paper [19] that allows solving dedicated engineering problem of process designing.

\section{Materials and Methods}

The design scheme of the application of ANN for directional research is presented in Fig. 1. This type of research is widely used for carrying out numerical simulations of mechanical and hydro-mechanical systems. It consists of three stages: mathematical modeling, numerical simulation, and experimental research; ANN learning process by the obtained experimental data; numerical evaluation of parameters.

The design scheme of the application of ANN for solving the inverse problem is presented in Fig. 2.
This type of research is used for providing the nonlinear regression procedure with the aim to evaluate unknown parameters of the mathematical model of the hydro-mechanical process. It includes the following stages: mathematical modeling, numerical simulation, and experimental research; ANN learning process by the obtained experimental data; identification of the hydro-mechanical system.

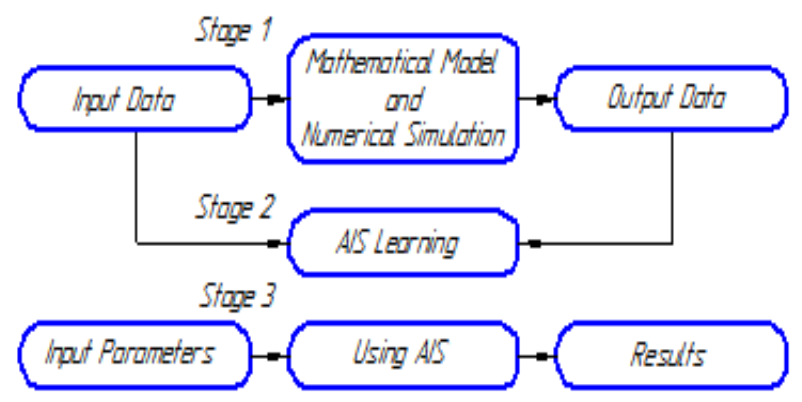

Figure 1: The design scheme of carrying out directional research using ANN.

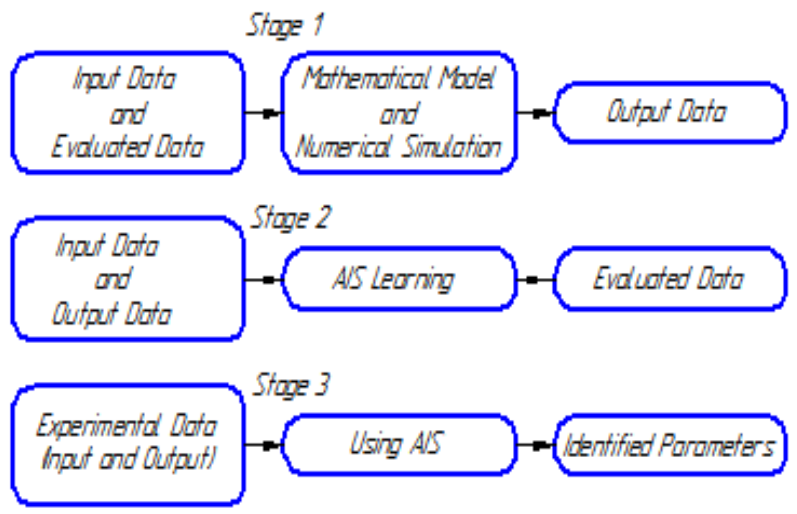

Figure 2: The design scheme of carrying out inverse research using ANN.

\section{Results and Discussion}

\section{- Estimation of the Separation Equipment operating Parameters}

The work of the most separation devices with heterogeneous systems as an operating environment is based on the inertia force impact due to its valuable influence on the gas-dispersed flow. At the same time, an important problem is the removal of the captured liquid films from the functional surfaces due to its contact with the turbulent gas flow. In this case, the critical flow rate, at which the secondary removal occurs, does not change significantly. Consequently, there is no significant extension of a range of effective operation for the related separation equipment. As a result, a fundamentally new method for the separation of heterogeneous systems has been developed that allows significantly extending this range. Moreover, related mathematical models were proposed for describing thermal and mass exchange processes in operating channels of the separation equipment. Particularly, 
equations of the movement of fine particles in a turbulent flow were found out for the channel with the equidistant curvilinear walls [20], equations describing the liquid film removal from the contact surfaces were determined [21], as well as deflections of the deformable contact surfaces were evaluated as a dependence on the flow parameters [22].

It should be additionally noted that the nontrivial approach for solving the problem of capturing a highly dispersed dropped fluid from a gas flow concerns a method of gas-dynamic separation. In this case, functional elements (Fig. 3 a) operate as an automatic control system with spring forces as a control action and hydraulic resistance as a regulated object. Increasing the flow rate leads to an increase of the dynamic pressure of the gas-liquid flow and internal bending stress of the deformable plate. As a result, plate deflection and hydraulic diameter are increased. Consequently, the coefficient of hydraulic losses is also decreased.

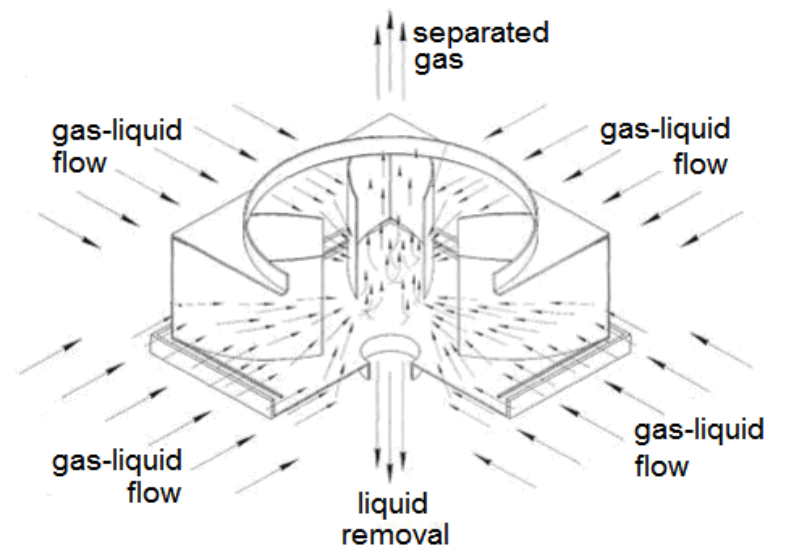

(a)

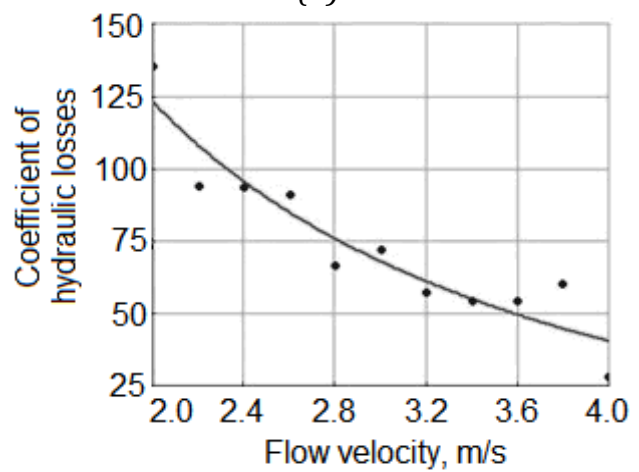

(b)

Figure 3: The design scheme of the vibration-inertial separation device (a) and dependence between the coefficient of hydraulic losses and flow velocity (b).

As a result of numerical simulation of aeroelastic interaction between flexible elements of the vibration-inertial separation device with a gas-liquid flow, the dependence of the coefficient of hydraulic losses $\zeta$ on the flow velocity $\mathrm{v}$ can be determined using the following expression (Fig. $3 \mathrm{~b}$ ):

$$
\zeta=\frac{2 \Delta p(v)}{\rho_{m} v^{2}},
$$

$\rho_{m}$ - density of a gas-liquid mixture;

$\Delta p(v)$ - pressure difference as a nonlinear analytically undetermined function of the flow velocity $v$.

For choosing the analytical function, which properly approximates the obtained data, the power, exponential, logarithmic, hyperbolic, fractional and rational dependencies were used. As a result, it is proved that dependence (1) can be approximated using the hyperbolic function. However, due to the impossibility to precise prediction of this data using the regression procedure, ANN should be implemented into these models for solving the problem of parameter identification for the abovementioned hydro-mechanical system.

The design schemes of the application of ANN for dynamic analysis of the hydro-mechanical system "multiphase flow - flexible elements" in the vibration-inertial separation device, particularly for evaluating the coefficient of hydraulic losses are presented in Fig. 4.

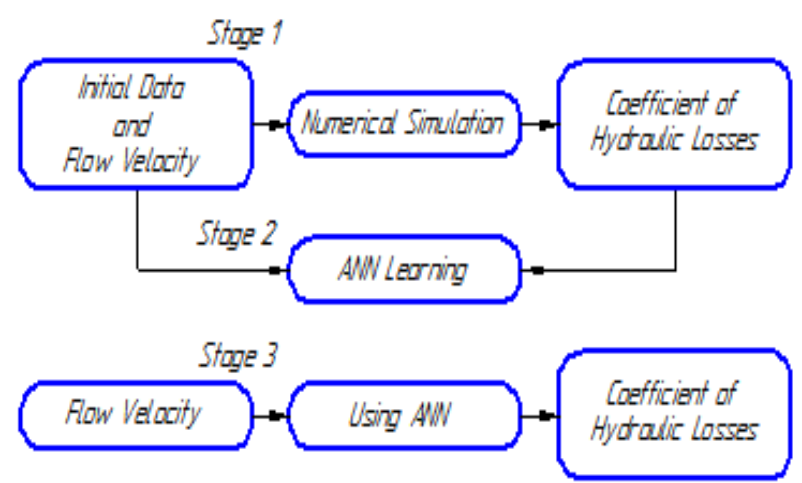

Figure 4: The design scheme of the application of ANN for evaluating the coefficient of hydraulic losses.

- Intensification of Clamping and Cutting Forces during Workpiece Machining in a Fixture

The main direction of increasing the efficiency of workpieces machining in multi-product manufacturing is the automation of manufacturing processes. An increase of automation process occurs due to the use of $\mathrm{CNC}$ machine tools and rapid adjustable process within the similar technological characteristics of the flexible fixtures. In this case, the following mutually contradictory conditions should be satisfied in fixture design: high accuracy depends on the rigidity; high stiffness of a fixture is ensured by increasing dimensions of its functional elements; increasing the fixture dimensions leads to an increase of metal consumptions; unacceptable increasing metal consumptions leads to cost growth of the fixture and related manufactured parts.

The design scheme of the adjustable fixture for workpiece machining is presented in Fig. 5. 


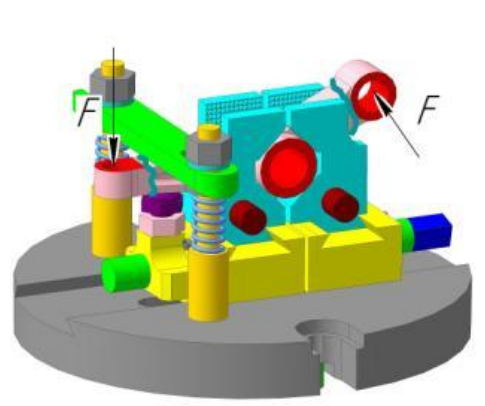

(a)

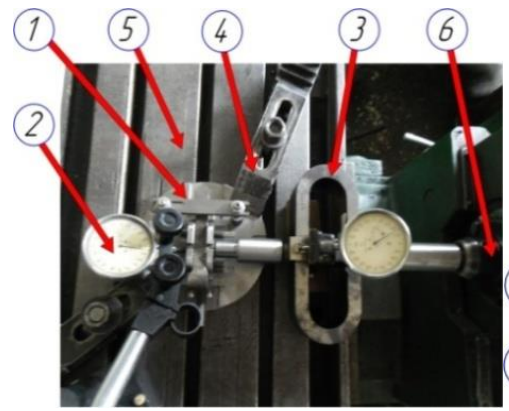

(b)

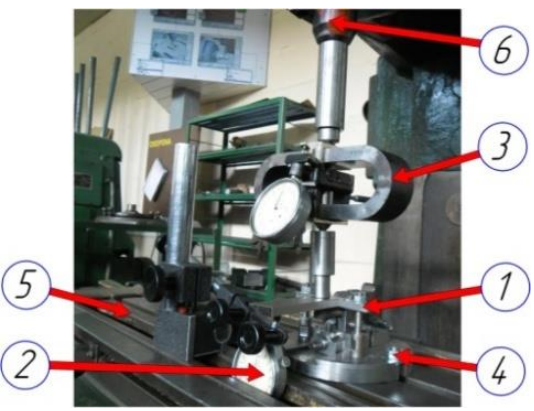

(c)

Figure 5. The design scheme (a) and experimental stand in horizontal (a) and vertical (c) planes for investigating the mechanical system "fixture - workpiece" [23]: 1 - fixture; 2 - indicator;

3 - dynamometer; 4 - fasteners; 5 - machine tool table; 6 - mandrel.

A general approach for the numerical simulation of the system "fixture - work-piece" is proposed in the work [24] on the example of lever machining. For this case study, ANN can be useful for determining an unknown system of cutting and clamping forces [17].

The mathematical model of the impact of clamping and cutting forces on deformations of functional elements can be written in a matrix form:

A proper appearance of the paper will help the reader go through it more easily. To obtain it is imperative that authors use this template.

$$
\left.\{U\}_{0}+\{\Delta U\}=[A]_{0}\{F\}_{0}+[\Delta A]_{\{F}\right\}_{d},
$$

$\{F\}_{0},\{F\}_{d}$ - column vector of generalized static loads (clamping forces and stationary parts of cutting forces) and dynamic loads (nonstationary parts of cutting forces);

$\{U\}_{0},\{\Delta U\}$ - column vectors of generalized static and dynamic displacements;

$[A] 0,[\Delta A]$ - compliance and auxiliary compliance matrices, elements of which $\mathrm{A}_{\mathrm{i}, \mathrm{j}}$ and $\Delta \mathrm{A}_{\mathrm{i}, \mathrm{j}}$ are determined by the numerical simulation data using the following expressions:

$$
A_{i, j}=\frac{U_{0}^{<i>}}{F_{0}^{<j>}} ; \Delta A_{i, j}=\frac{\Delta U_{i}}{F_{d}^{<j>}},
$$

$U_{0}^{<i>}$ - static displacement of $i$-th functional element due to the action of $j$-th unit generalized clamping force $F_{0}^{<j>}=1$;

$\Delta U^{i>}$ - dynamic displacement of $i$-th functional element due to the action of $j$-th unit generalized cutting force $F_{d}^{<j>}=1$.

The equation (2) can be solved analytically under the condition of linearity when the superposition principle can be applied. In this case, clamping and cutting forces are determined by the following dependencies:

$$
\begin{aligned}
& \{F\}_{0}=[A]_{0}^{-1}\{U\}_{0} ; \\
& \{F\}_{d}=[\Delta A]^{-1}\{\Delta U\}=[\Delta A]^{-1}\left\langle\{U\}-\{U\}_{0}\right\rangle,
\end{aligned}
$$

$\{U\}=\{U\}_{0}+\{\Delta U\}-$ column vector of total displacements.

Modern CAE systems allow implementing automated optimization tools for providing a rapid and accurate calculation of deflected mode, as well as for carrying out modal and harmonic analysis of fixtures. Thus, all the elements of the compliance matrices (3) can be determined numerically or experimentally.

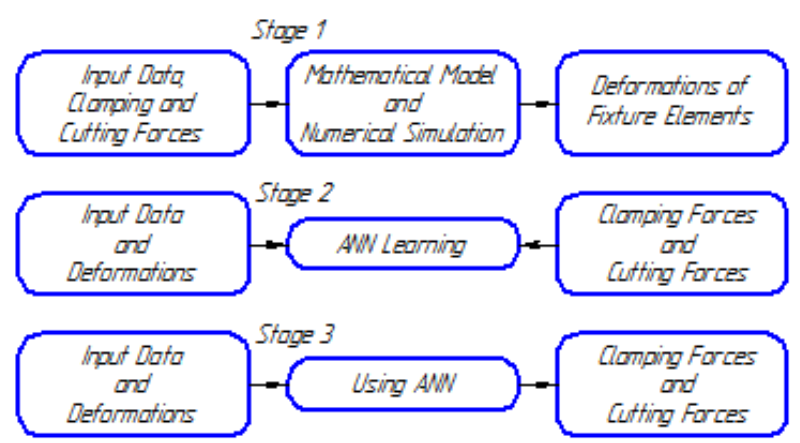

Figure 6. The design scheme of the application of ANN for evaluating clamping and cutting forces during workpiece machining in a fixture.

In the case of significant nonlinearities of the system "fixture - workpiece", the abovementioned analytical approach allows evaluating the first approximation only. Nevertheless, more precise solutions can be obtained by using ANN. The related design schemes of the application of ANN for evaluating clamping and cutting forces during workpiece machining in a fixture is presented in Fig. 6.

\section{- Dynamic Analysis of the Centrifugal} Machine's Rotor

Modern numerical approaches for the simulation of rotor dynamics are mainly based on the use of FEM analysis [25]. However, analytical research based on simplified discrete or continuous models considers Kirchhoff's hypothesis. Despite this fact, relatively large machine time is required for using 3D finite element models in CAE systems. Such software does not consider available nonlinearities in rotary systems. Thus, extending the opportunities of CAE programs by developing the related built-in applications is the significant problem to solve [26].

There are many research papers are devoted to solving the problems in the field of rotor dynamics. 
Particularly, using up-to-date computational software for evaluating natural frequencies based on the 2D model is proposed in the paper [27]. The method of parameter identification of unbalances is presented in the research work [28]. The influence of the deformable elements on rotor dynamics is researched in the paper [29]. The research work [30] is aimed at developing the approach for identifying resonance modes for rotary systems. The methods of ensuring experimental research of rotor dynamics are stated in the papers [31, 32]. Finally, the methodology solving nonlinear problems of rotor dynamics is proposed in the research paper [33].

The system of differential equations describing rotor dynamics can be transformed to the following matrix equation [34]:

$$
\left([C(\omega)]-\omega^{2}[M]\right)\{Y\}=\{F\},
$$

$\{F\},\{Y\}$ - amplitude column vectors of forces and displacements;

$[C(\omega)],[M]$ - global stiffness and inertia matrices; $\omega$ - frequency, which can be presented in the following polynomial dependence [35]:

$$
c(\omega)=c_{0}+\alpha \omega+\beta \omega^{2},
$$

parameters $c_{0}, \alpha, \beta$ of which are needed to be evaluated.

In case of free oscillations $\{F\}=\{0\}$, critical frequencies $\{\Omega\}$ of the rotor are determined from the following nonlinear equation [36, 37]:

$$
\operatorname{det}\left([C(\Omega)]-\Omega^{2}[M]\right)=0 .
$$

On the example of the multistage centrifugal compressor 295GC2-190/44-10M on magnetic bearings with operating rotor speed in the range $3710-5565 \mathrm{rpm}$ and power capacity about $17 \mathrm{MW}$ as a part of the gas pumping unit GPU-C-16/102-3.32M produced by the Public Joint Stock Company "Sumy Machine-Building Science - and - Production Association", the abovementioned technique allows evaluating the following critical frequencies and parameters of the dependence (2): $\Omega_{1}=117 \mathrm{rad} / \mathrm{s}$, $\Omega_{2}=264 \mathrm{rad} / \mathrm{s}, \Omega_{3}=513 \mathrm{rad} / \mathrm{s} ; c_{0}=21.8 \cdot \mathrm{MN} / \mathrm{m}$, $\alpha=-6130 \mathrm{~N} \cdot \mathrm{s} / \mathrm{m}, \beta=180 \mathrm{~N} \cdot \mathrm{s}^{2} / \mathrm{m}$ (Fig. 7).

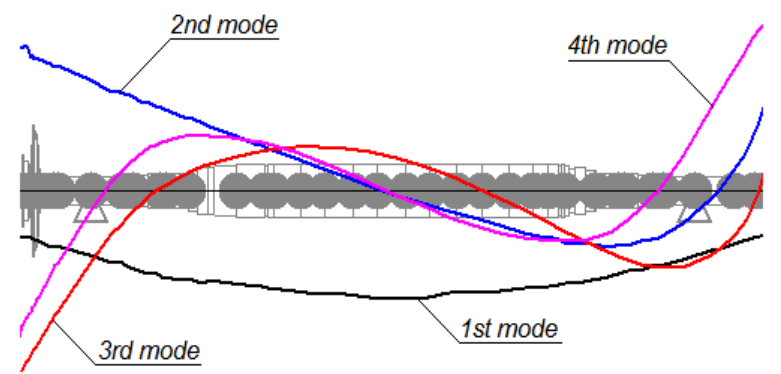

Figure 7. Design model and modes shapes of the compressor's rotor [18].

The abovementioned model can be also realized using ANN for determining critical frequencies in the case of a nonlinear mathematical model. The design schemes of the application of ANN for the determination of critical frequencies of the rotor, as well as for the evaluation of bearing stiffness coefficients are presented in Fig. 8, which shows the procedure for identification of rotor bearing stiffness characteristic by combined using a finite element model of rotor dynamics and ANN.

"Visual Gene Developer" software allows solving the stated problem using genetic algorithms and artificial neural networks and. Moreover, it provides a graphical visualization of ANN training procedure [17], which is shown in Fig. 9.

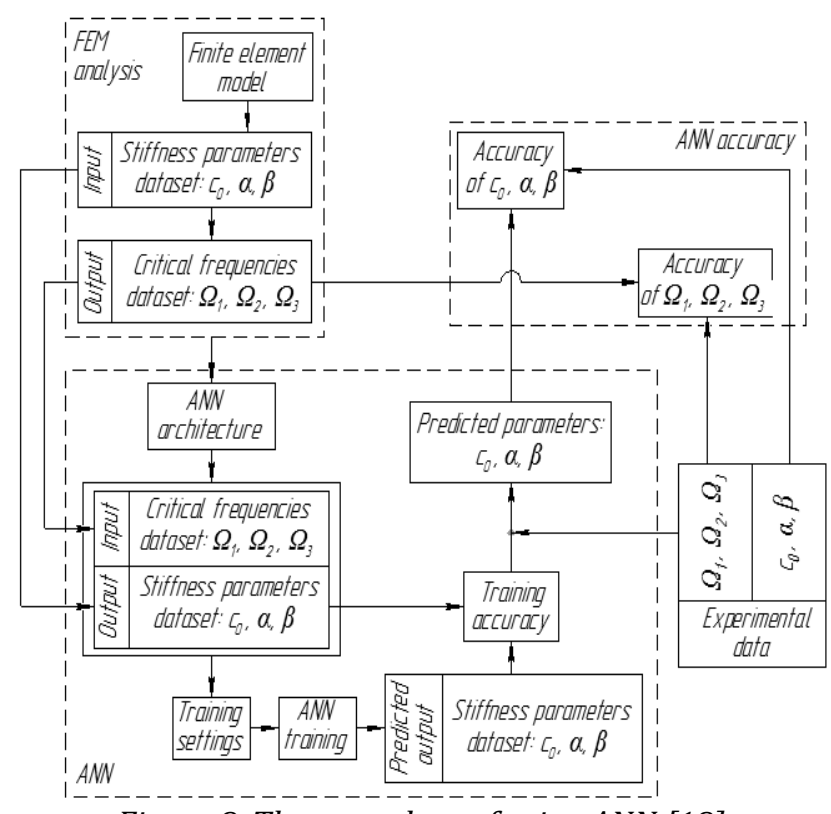

Figure 8. The procedure of using ANN [18]. 
The number of layers and the distribution of neurons in layers is determined by the condition of full-time operating of all neurons. Decreasing the number of hidden layers and corresponding neurons leads to decreasing accuracy of the subsequent evaluation of rotor bearing stiffness characteristics, while an unreasonable increasing of neurons and layers leads to increasing the learning time and to non-involved neurons.

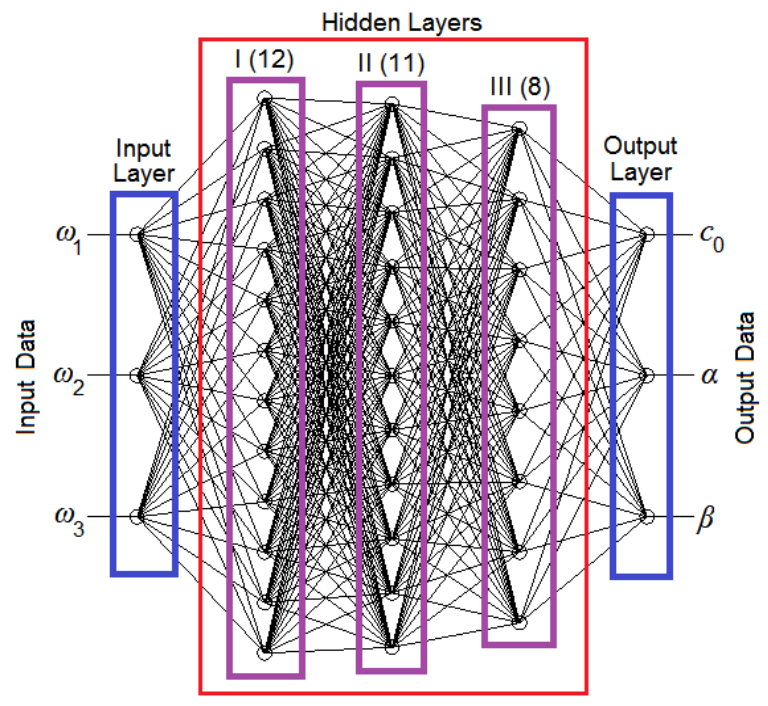

a

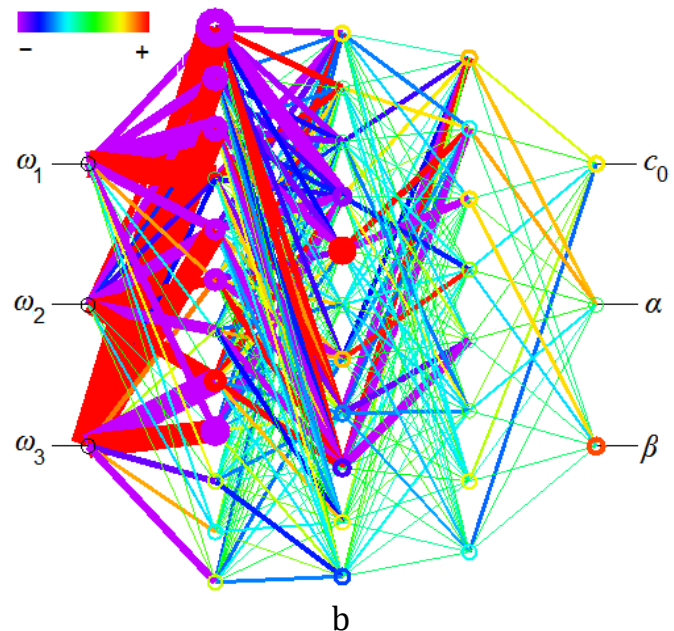

Figure 8. ANN architecture (a) and map analysis (b).

The approach is realized for the following settings: total number of neurons - 31; number of hidden layers - 3; learning rate - 0.001; transfer function - hyperbolic tangent; total number of training cycles - 106; target error - 1.10-5; initialization method of threshold - random; initialization of weight factor - random; update interval - 500 cycles; maximum relative error of the evaluated results -0.009 .

As a result of using ANN, the following parameters are evaluated: $\Omega_{1}=118 \mathrm{rad} / \mathrm{s}, \Omega_{2}=256$ $\mathrm{rad} / \mathrm{s}, \Omega_{3}=511 \mathrm{rad} / \mathrm{s} ; c_{0}=24.6 \cdot \mathrm{MN} / \mathrm{m}, \alpha=-2628$ $\mathrm{N} \cdot \mathrm{s} / \mathrm{m}, \beta=200 \mathrm{~N} \cdot \mathrm{s} 2 / \mathrm{m}$.
The actual parameters obtaining as a result of rotor balancing procedure

$\left(\Omega_{1}=117 \mathrm{rad} / \mathrm{s}, \Omega_{2}=256 \mathrm{rad} / \mathrm{s}, \Omega_{3}=511 \mathrm{rad} / \mathrm{s} ; c_{0}\right.$ $=24.5 \cdot \mathrm{MN} / \mathrm{m}, \alpha=-2900 \mathrm{~N} \cdot \mathrm{s} / \mathrm{m}$, and $\beta=209 \mathrm{~N} \cdot \mathrm{s} 2 / \mathrm{m}$ ) confirm the need of using ANN for determining parameters of the mechanical system "rotor bearing supports" due to the possibility to minimize the maximum relative error. However, the direct use of the regression procedure allows accurately determining the critical frequencies only.

\section{Conclusions}

The generalized research methodology for implementing artificial neural networks is proposed for solving applied problems in the field of mechanical, manufacturing and chemical engineering. The related design schemes are proposed for parameter identification of mathematical models of the following relationships between the mechanical and hydro-mechanical parameter: "rotor dynamic - bearing stiffness", "deformations of the fixture - clamping and cutting forces", and "flow rate - coefficient of hydraulic losses". This methodology is aimed at solving the direct and inverse hydro-mechanical problems based on the combined application of an artificial neural network, analytical dependencies, and numerical simulations.

The design schemes of the application of an artificial neural network for dynamic analysis of the hydro-mechanical system "multiphase flow - flexible elements" in the vibration-inertial separation device is presented on the example of parameter identification of the coefficient of hydraulic losses.

The ways for increasing the efficiency of workpiece machining in multiproduct manufacturing are proposed. Additionally, the comprehensive approach for combined using AIS, numerical simulation and mathematical modeling is developed for the case of parameter identification of the mechanical system "fixture - workpiece".

Since the problem of evaluation of stiffness characteristics for rotor bearings with-in the dependence of critical frequencies of the rotor has not been solved previously, using the artificial neural network for evaluating parameters of the approximating curve "bearing stiffness - rotor speed" by the numerical simulation results is a novel approach. It should be noted that the use of artificial intelligence systems allows significantly increasing the accuracy of parameter evaluation in comparison with the regression procedures.

Please observe the following rules: pages should have the following format: A4 paper, Cambria font, and the following margin settings: header: $1,5 \mathrm{~cm}$, footer: $1,5 \mathrm{~cm}$, bottom: $1,5 \mathrm{~cm}$, top: $1,5 \mathrm{~cm}$, inside: 2,5 $\mathrm{cm}$ and outside: $1,5 \mathrm{~cm}$. 


\section{Acknowledgments}

The proposed models were designed within the research project "Development and implementation of energy efficient modular separation devices for oil and gas purification equipment" (No. 0117U003931) ordered by the Ministry of Education and Science of Ukraine. The numerical simulation results were obtained due to the close collaboration between the Faculty of Technical Systems and Energy Efficient Technologies of Sumy State University (Ukraine) and the Faculty of Mechanical Engineering and Management of Poznan University of Technology (Poland).

\section{References}

[1] Fuks, K., Kawa, A., Wieczerzycki, W.: Improved esourcing strategy with multi-agent swarms. In: Computational Intelligence for Modelling Control and Automation, pp. 488-493 (2008).

[2] Kunz, G., Machado, J., Perondi, E.: Using timed automata for modeling, simulating and verifying networked systems controller's specifications. In: Neural Computing and Applications, vol. 28(5), pp. 1031-1041 (2017).

[3] Santos, A.S., Varela, M.L.R., Putnik, G.D., Madureira, A.M.: Alternative Approaches Analysis for Scheduling in an Extended Manufacturing Environment. In: Proceedia of Nature and Biologically Inspired Computing, pp. 97-102 (2014).

[4] Sika, R., Rogalewicz, M.: Methodologies of knowledge discovery from data and Data Mining methods in mechanical engineering. In: Management and Production Engineering Review, vol. 7 (4), pp. 97-108 (2016).

[5] Dostatni, E., Diakun, J., Grajewski, D., Wichniarek, R., Karwasz, A.: Multi-agent System to Support Decision-Making Process in Ecodesign. In: Advances in Intelligent Systems and Computing, vol 368, pp. 463-474 (2015).

[6] Chandima, R.M., Antosz, K.: Development of a Risk Matrix and Extending the Risk-based Maintenance Analysis with Fuzzy Logic. In: Procedia Engineering, vol. 182, pp. 602-610 (2017).

[7] Zhao, L., Li, W., Geng, L., Ma, Y.: Artificial Neural Networks Based on Fractal Growth. Advances in Automation and Robotics, vol. 2, pp. 323-330 (2011).

[8] Ferraz, A., Brito, J., Carvalho, V., Machado, J.: Blood Type Classification Using Computer Vision and Machine Learning. In: Neural Computing and Applications, vol. 28, pp. 2029-2040 (2017).

[9] Putnik, G.D., Ferreira, L., Shah, V., Putnik, Z., Castro, H., Cruz-Cunha, M.M., Varela, L.: Effective Service Dynamic Packages for Ubiquitous Manufacturing System. In.: Virtual and
Networked Organizations, Emergent Technologies and Tools, pp.207-219 (2011).

[10] Varela, M.L.R., Ribeiro, R.A.: Distributed Manufacturing Scheduling Based on a Dynamic Multi-criteria Decision Model. In: Recent Developments and New Directions in Soft Computing. Studies in Fuzziness and Soft Computing, vol. 317, pp. 81-93 (2014).

[11] Li, F., Zhang, K.: A Genetic Algorithm-Based Quasi-Linear Regression Method and Application. In: Proceedings of the 2nd International Symposium on Computer, Communication, Control and Automation, ISCCCA-13, pp. 438-441 (2013).

[12] Li, F.-C., Yang, K.: Research of the Regression Method Based on Quasi-linear Function. In: 2010 International Conference on Web Information Systems and Mining, vol. 2, 11794542 (2010), doi: 10.1109/WISM.2010.36.

[13] Li., F., Jin, C., Shi, Y., Yang, K.: Study on quasilinear regression methods. In: International journal of innovative computing, information and control, IJICIC 2012, vol. 8(9), pp. 6259-6270 (2012).

[14] Tholerus, S., Hellsten, T., Johnson, T.: Comparisons of the nonlinear and the quasilinear model for the bump-on-tail instability with phase decorrelation. In: Journal of Physics: Conference Series, vol. 561, 012019 (2014), doi: 10.1088/1742-6596/561/1/012019.

[15] Pelikan, M.: Probabilistic model building Genetic Algorithm (PMBGA): A survey. In: Proceedings of the 13th Annual Conference Companion on Genetic and Evolutionary Computation, GECCO 2011, pp. 913-940 (2011).

[16] Kapania, R.K., Liu, Y.: Applications of Artificial Neural Networks in Structural Engineering with Emphasis on Continuum Models. In: Virginia Polytechnic Institute and State University, Blacksburg (1998).

[17] Pavlenko, I., Trojanowska, J., Ivanov, V., Liaposhchenko, O.: Scientific and methodological approach for the identification of mathematical models of mechanical systems by using artificial neural networks. In: Machado J., Soares F., Veiga G. (eds) Innovation, Engineering and Entrepreneurship. HELIX 2018. Lecture Notes in Electrical Engineering, vol. 505, pp. 299-306 (2019), doi: 10.1007/978-3-319-91334-6_41.

[18] Pavlenko, I., Simonovskiy, V., Ivanov, V., Zajac, J., Pitel, J.: Application of Artificial Neural Network for Identification of Bearing Stiffness Characteristics in Rotor Dynamics Analysis. In: Ivanov V. et al. (eds.) Advances in Design, Simulation and Manufacturing. DSMIE-2018. Lecture Notes in Mechanical Engineering, pp. 325-335 (2019), doi: 10.1007/978-3-31993587-4_34.

[19] Lazarevska, M., Knezevic, M., Cvetkovska, M., Trombeva-Gavrilovska, A.: Application of 
artificial neural networks in civil engineering. In: Tehnicki Vjesnik, vol. 21(6), pp.1353-1359 (2014).

[20] Liaposhchenko, O., Pavlenko, I., Nastenko, O.: The model of crossed movement and gas-liquid flow interaction with captured liquid film in the inertial-filtering separation channels. In: Separation and Purification Technology, vol. 173, pp. 240-243 (2017), doi: 10.1016/j.seppur.2016.08.042.

[21] Liaposhchenko, O.O., Sklabinskyi, V.I., Zavialov, V.L., Pavlenko, I.V., Nastenko, O.V., Demianenko, M.M: Appliance of Inertial Gas-Dynamic Separation of Gas-Dispersion Flows in the Curvilinear Convergent-Divergent Channels for Compressor Equipment Reliability Improvement. In: IOP Conference Series: Materials Science and Engineering, vol. 233, 012025 (2017), doi: 10.1088/1757-899X/233/1/012025.

[22] Sklabinskyi, V., Liaposhchenko, O., Pavlenko, I., Lytvynenko, O., Demianenko, M.: Modelling of liquid's distribution and migration in the fibrous filter layer in the process of inertial-filtering separation. In: Ivanov V. et al. (eds.) Advances in Design, Simulation and Manufacturing. DSMIE2018. Lecture Notes in Mechanical Engineering, pp. 489-497 (2019), doi: 10.1007/978-3-31993587-4_51.

[23] Ivanov, V., Dehtiarov, I., Denysenko, Y., Malovana, N., Martynova, N.: Experimental diagnostic research of fixture. In: Diagnostyka, vol. 19(3): pp. 3-9 (2018), doi: 10.29354/diag/92293.

[24] Ivanov, V., Mital, D., Karpus, V., Dehtiarov, I., Zajac, J., Pavlenko, I., Hatala, M.: Numerical simulation of the system "fixture-workpiece" for lever machining. In: International Journal of Advanced Manufacturing Technology, vol. 91(14), pp. 79-90 (2017), doi: 10.1007/s00170-0169701-2.

[25] Swanson, E, Powell, C D, Weissman, S.: A practical review of rotating machinery critical speeds and modes. In: Sound and Vibration, vol. 39(5), pp. 10-17 (2005).

[26] Sika, R, Hajkowski, J: Synergy of modeling processes in the area of soft and hard modeling. Proceedings of 8th International Conference on Manufacturing Science and Education (MSE 2017), Trends in new industrial revolution, Sibiu, MATEC Web of Conference 121, 04009 (2017), doi: 10.1051/matecconf/2011721105007.

[27] Muminovic, A.J., Braut, S., Muminovic, A., Saric, I.: Numerical and analytical analysis of elastic rotor natural frequency. In: Technology, Education, Management, Informatics, vol. 3(4), pp. 323-328 (2014).
[28] Wang, A., Cheng, X., Meng, G., Xia, Y., Wo, L., Wang, Z.: Dynamic analysis and numerical experiments for balancing of the continuous single-disc and single-span rotor-bearing system. In: Mechanical Systems and Signal Processing, vol. 86, pp. 151-176 (2017).

[29] Villa, C., Sinou, J., Thouverez, F.: Stability and vibration analysis of a complex flexible rotor bearing system. In: Communications in Nonlinear Science and Numerical Simulation, vol. 13(4), pp. 804-821 (2008).

[30] Bai, C., Zhang, H., Xu, Q.: Subharmonic resonance of a symmetric ball bearing-rotor system. In: International Journal of Non-Linear Mechanics, vol. 50, pp. 1-10 (2013).

[31] Larsen, T.J., Kim, T.: Experimental and numerical study of a new dynamic phenomenon for two-bladed wind turbines. In: Proceedings of the 25th International Ocean and Polar Engineering Conference, pp. 547-553 (2015).

[32] Ocampo, J.C., Wing, E.S.G., Moroyoqui, F.J.R., Pliego, A.A., Ortega, A.B., Mayen, J.: A novel methodology for the angular position identification of the unbalance force on asymmetric rotors by response polar plot analysis. In: Mechanical Systems and Signal Processing, vol. 95, pp. 172-186 (2017).

[33] Sinou, J., Didier, J., Faverjon, B.: Stochastic nonlinear response of a flexible rotor with local nonlinearities International. In: Journal of Non-Linear Mechanics, vol. 74, pp. 92-99 (2015).

[34] Pavlenko, I.: Static and dynamic analysis of the closing rotor balancing device of the multistage centrifugal pump. In: Applied Mechanics and Materials, Trans Tech Publications, vol. 630, pp. 248-254 (2014), doi: 10.4028/www.scientific.net/AMM.630.248.

[35] Pavlenko, I.V., Simonovskiy, V.I., Demianenko, M.M.: Dynamic analysis of centrifugal machines rotors supported on ball bearings by combined application of 3D and beam finite element models. In: IOP Conference Series: Materials Science and Engineering, vol. 233, pp. 1-8, 012053 (2017), doi: 10.1088/1757899X/233/1/012053.

[36] Reddy, M C S, Sekhar, A S: Application of artificial neural networks for identification of unbalance and looseness in rotor bearing systems. International Journal of Applied Science and Engineering 11(1), pp. 69-84 (2013).

[37] Altaf, S., Mehmood, M.S., Imran, M: Implementation of efficient artificial neural network data fusion classification technique for induction motor fault detection. Journal of Engineering Sciences 5(2), pp. E16-E21 (2018), doi: $10.21272 /$ jes.2018.5(2).e4. 